\title{
ESTUDIO DE LOS HONGOS QUE FRUCTIFICAN EN LA VEGETACION RELICTA DE ABIES PINSAPO BOISS., EN ESPAÑA PENINSULAR. I. ASCOMYCOTINA
}

\author{
J.M. LARIOS, M. HONRUBIA \& G. MORENO
}

\begin{abstract}
RESUMEN: Se citan veinte táxones especificos de la subdivisión Ascomycotina, recolectados en los pinsapares de las provincias de Cádiz y Málaga. De entre ellos, cabe destacar la presencia de: Ascocalyx asiaticus Groves, Coniochaeta malacotricha (Auersw.) Traverso, Lophiostoma alpigenum Fuckel, Ophiobolus erythrosporus (Riess) Wint., Rhamphoria obliqua Karst., Scoleconectria cucurbitula (Tode: Fr.) Booth y Zignoëlla fallax Saccardo. Se comentan las principales características morfológicas y anatómicas y se critica la posición taxonómica de las especies polémicas. Se ilustran, con figuras originales, los caracteres más destacables de las especies más interesantes.
\end{abstract}

SUMMARY: Study on the fungi that grow in the relict vegetation of Abies pinsapo Boiss. in the Spanish Peninsula. I. Ascomycotina. Twenty specific taxa of the subdivision Ascomycotina, collected in Spanish fir woods in the province of Cádiz and Málaga are cited. Among them, the presence of Ascocalyx asiaticus Groves, Coniochaeta malacotricha (Auersw.) Traverso, Lophiostoma alpigenum Fuckel, Ophiobolus erythrosporus (Riess.) Wint., Rhamphoria obliqua Karst., Scoleconectria cucurbitula (Tode: Fr.) Booth and Zignoëlla fallax Saccardo, should be pointed out. The most interesting morphology and anatomic characteristics are commented on and the taxonomic position of the problematic species is critized. The most oustanding characteristics of the most interesting species are illustred with the original plates.

\section{INTRODUCCION}

Los pinsapares ibéricos siempre han despertado el interés de botánicos y naturalistas hispanos y extranjeros. Se conocen gran número de datos referentes a la flora vascular y vegetación de las zonas ocupadas y limítrofes de Abies pinsapo Boiss.

Sin embargo, son escasos los estudios realizados exclusivamente sobre el componente fúngico de los pinsapares de Cádiz y Málaga. La mayor parte de los datos disponibles al respecto están dispersos en la bibliografía, en trabajos de indole más general. Es el caso de los artículos de Malençon (1968) y Bertault (1974) sobre la flora micológica andaluza, de Malençon \& Bertault (1976) sobre los hongos de la Península Ibérica, de Benito \& Torres (1965) sobre enfermedades de las coniferas españolas, etc.

Sólamente hemos podido constatar el trabajo de Manjon \& Moreno (1983) donde se estudian exclusivamente, Aphyllophorales de los pinsapares ibéricos. 
Esta precariedad de datos y, sobre todo, ante el actual estado de los pinsapares españoles, con indice de mortalidad elevado, nos condujo a iniciar un proyecto de investigación sobre "Estudio de los hongos que fructifican en la vegetación relicta de Abies pinsapo Boiss., en España Penínsular", subvencionado por la CAICYT.

Se ofrece, en este artículo, un avance de los primeros resultados que, sobre Ascomycotina, se han obtenidos en el desarrollo de dicho proyecto.

Se citan un total de 20 especies. Se ordenan según los criterios taxonómicos propuestos por Hawksworth \& col. (1983). Se critica la posición taxonómica de algunas de las especies polémicas. Para cada una de las citas dadas, se indica: localidad de procedencia del material estudiado, recolector (expresado por las iniciales de los autores del artículo), fecha de recolección y número de registro. El material estudiado está depositado en la Mycotheca del Herbario MUB (Universidad de Murcia) registro bajo las iniciales JL, con el correspondiente número de registro, disponible para cualquier consulta o posterior revisión.

\section{CATALOGO}

\section{DIAPORTHALES}

Rhamphoria obliqua Karsten ss. Berlese

Material estudiado: Sierra de las Nieves, Ronda (Málaga). Leg.- J.L. \& M.H., 13.XI.1983 (JL 7060). (Fig. 1: A-B-C).

Hábitat: Sobre rama muerta descortezada de Abies pinsapo.

Observaciones: Especie fácil de reconocer por sus peritecios tumbados, con papila lateral, curvada y ostiolos verticales. El material estudiado coincide con la descripción dada por Berlese (1968) para este taxon.

El género Rhamphoria Niessl., ha sido incluido por la mayoría de los autores modernos, dentro del orden Diaporthales, en la familia Diaporthaceae (von Arx \& Müller, 1954; Müller \& von Arx, 1962, 1973; Munk, 1953, 1957). Otros autores son partidarios de colocar este género en la familia Ceratostomaceae (Luttrell, 1951; Chadefaud, 1960 in Barr, 1978), dentro del mismo orden.

Barr (1978) considera que "pese a presentar algunos caracteres de Diaporthales, como centrum pseudoparenquimático y ascos con ani110 apical, el hábitat y la pared carbonácea no son típicos de este orden". Por estas razones, dispone el género Rhamphoria, en la familia Lasiosphaeriaceae, en el orden Sordariales. Hawksworth \& al. (1983) incluyen el taxon de Niessl en el orden Diaporthales, sin asignarle una familia determinada.

\section{Valsa friesii (Duby) Fuckel}

Material estudiado: Pinsapar del Cortijo de la Nava de San Luis, Ronda (Málaga). Leg.- J.L., 22.XI.1984 (JL 7075, JL 7088).

Hábitat: Sobre rama muerta de Abies pinsapo.

Observaciones: El material estudiado coincide con las descripciones dadas para este taxon por Munk (1957) y Ellis \& Everhart (1892). Solamente se aprecia una ligera diferencia en el diámetro de las as-

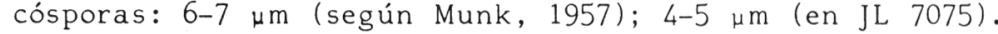

Valsa friesii presenta una alta especificidad respecto al sus- 
trato. Ha sido hallada siempre sobre diversas especies de abetos: Abies pectinata (Munk, 1957), A. balsamea (Ellis \& Everhat, 1892).

\section{DIATRYPALES}

Eutypa flavovirescens (Hoffm.) Tulasne

(E. flavovirens (Hoffm.) Tulasne ss. Munk)

Material estudiado: Sierra de el Pinar, Grazalema (Cádiz). leg. M.H., 14.XI.1983 (JL 7041, JL 7042); 14.VI.1984, (JL 7086). Pinsapar del Cortijo de la Nava de San Luis, Ronda (Málaga). Leg. J.L., 22.XI.1984 (JL 7085). (Fig. 1:D).

Hábitat: Sobre madera muerta, descortezada, de Quercus rotundifolia y Hedera helix.

Observaciones: A pesar de su variabilidad morfológica externa, es fácil de reconocer, como indica Munk (1957), por el color verde-amarillento del pseudostroma.

Es estado anomorfo corresponde a Cytosporina flavo-virescens (Sacc.) Trav., que presenta conidios alantoides, hialinos, de 25-30 $\times 1-2,5 \mu \mathrm{m}$.

Eutypa polymorpha (Nits.) Sacc. subsp. cyclospora (Nits.) Sacc.

Material estudiado: Pinsapar de la Sierra de las Nieves, Yunquera (Málaga). Leg. J.L., 23.XI.1984 (JL 7084). (Fig. 1: E-F).

Hábitat: Sobre madera descortezada, en descomposición de Abies pinsapo.

Observaciones: Especie de morfología variable. El taxon subespecífico se reconoce fácilmente por sus ascósporas muy curvadas y de menor tamaño que las del tipo (Berlese, 1968).

\section{DOTHIDEALES}

Hysterographium fraxini (Pers.) De Notaris

Material estudiado: Subida a la Sierra de las Nieves, Ronda (Málaga). Leg. M.H., 13.XI.1983 (JL 7047, JL 7063).

Hábitat: Sobre ramas muertas de acebuche (Olea europaea var. sylvestris) y de Rhamnus oleoides.

Observaciones: Especie banal, recientemente citada para el S.E. ibérico (Honrubia \& al., en prensa).

Lophiostoma alpigenum Fuck.

(Lophiotrema alpigenum (Fuck.) Sacc.)

Material estudiado: Pinsapar de la Nava de San Luis, Ronda (Málaga). Leg. J.L., 22.XI.1984 (JL 7079). (Fig. 1: G).

Hábitat: Sobre rama muerta de Quercus rotundifolia.

Observaciones: El material estudiado por nosotros responde bien a la descripción dada por Saccardo (1893) para Lophiotrema alpigenum, aunque presenta ascósporas con $7-9$ septos transversales. Chester \& Bell (1970) indican la presencia de hasta 13 septos transversales en ascósporas maduras. Por otra parte, ninguno de estos autores subraya la existencia de vaina gelatinosa en las ascósporas jóvenes.

Distintos autores (Chester \& Bell, 1970; von Arx \& Müller, 1975 
y Hawksworth \& col., 1983), consideran el género Lophiotrema Sacc. como sinónimo de Lophiostoma Ces. \& Not. Munk (1957), por el contrario, prefiere mantener como válido el rángo genérico de Lophiotrema, hasta contar con un más detallado estudio del género. Por nuestra parte, mantenemos los criterios indicados por Hawksworth \& col. (1983).

Lophium mytilinum (Pers.) Fr.

Material estudiado: Pinsapar del Cortijo de la Nava de San Luis, Ronda (Málaga). Leg. M.H., 13.XI.1984'(JL 7044). Sierra de El Pinar, Grazalema (Cádiz). Leg. G.M. \& J.L., 14.XI.1984 (JL 7049). Los Reales, Sierra Bermeja, Estepona (Málaga). Leg. J.L., 13.VI.1984 (JL 7053, JL 7055). (Fig. 2: A-B).

Hábitat: Sobre ramas muertas, descortezadas y madera de troncos abatidos, descortezados o no, de Abies pinsapo.

Observaciones: Se caracteriza por sus fructificaciones en forma de concha de mejillón o almeja, de 450-750 x 300-425 x 400-625 um y las ascósporas filiformes de $190-250 \times 0,8-1,5 \mu \mathrm{m}$, dispuestas paralelamente en el interior del asco. Ha resultado ser el taxon más constante sobre Abies pinsapo. Habiendo sido encontrado abundantemente en todos los pinsapares de Cádiz y Málaga. Malençon \& Bertault (1976) lo citan sobre Pinus pinaster en la Sierra del Oreganal (Málaga). Recientemente, Checa \& Moreno (1984) indican su presencia en la provincia de Guadalajara, sobre Pinus sylvestris.

Bertault (1974) cita, sobre Abies pinsapo, en el "pinar de San Cristobal", Lophium elatum Grev. Este taxon es transferido (Zogg, 1962) al género Glyphium Nitschke ex Lehmann, por sus fructificaciones erectas, verticalmente alargadas, en forma de cabeza de hacha.

Mytilidion tortile (Schw.) Ell. \& Ev.

Material estudiado: Sierra de las Nieves, Ronda (Málaga). Leg. J.L. \& M.H., 13.XI.1983 (JL 7048). Pinsapar del Cortijo de la Nava de San Luis, Ronda (Málaga). Leg. J.L., 22.XI.1984 (JL 7076). Sierra de El Pinar, Grazalema (Cádiz). Leg. J.L., 12.XI.1983 (JL 7081). (Fig. 2: C-D ).

Hábitat: Muy frecuente sobre ramas muertas, descortezadas, de Abies pinsapo.

Observaciones: Se caracteriza por sus fructificaciones subgregarias, de color negro, superficiales, con forma de concha de almeja y por sus ascósporas de 15-20(23) x 5-6,5 $\mu \mathrm{m}$, uniseriadas, elíptico-fusiformes, de color pardo, triseptadas, constrictas a nivel de los septos. Típica de coníferas, ha sido encontrado frecuentemente sobre Abies pinsapo, en casi la totalidad de las localidades prospectadas.

El material estudiado coincide con las descripciones dadas por Ellis \& Everhart (1892) y Zogg (1962), para este taxon. Por contra, Saccardo (1883) indica caracteres, referentes al tamaño de ascos y ascósporas, notablemente distintos.

Ophiobolus erythrosporus (Reiss) Winter

Material estudiado: Sierra de las Nieves, Ronda (Málaga). Leg. J.L., 13.Vi.1984 (JL 7069). (Fig. 3: A-B-C-D-E).

Hábitat: Sobre madera muerta no identificada.

Observaciones: La mayoría de los autores consultados (Saccardo, 1883 ; Berlese, 1968; Munk, 1957; Dennis, 1978, Sivanesan, 1984), in- 
cluyen, en el género Ophiobolus Riess, gran parte de Pleosporáceos con ascósporas filiformes o muy alargadas. Holm (1957) combina varias especies del género Ophiobolus a otros géneros (Leptosphaeria, Nodulosphaerica, Phaeosphaeria y Entodesmium). Considera Nodulosphaeria erythrospora (Riess) Holm (=Ophiobolus erythrosporus (Riess) Wint.). Según algunos autores como Holm (1957), el género Nodulosphaeria se caracteriza por presentar ascósporas filiformes con célula mediana superior de mayor diámetro que las restantes. Siguiendo a Munk (1957) y Dennis (1978), consideramos este taxon como Ophiobolus erythrosporus (Riess) Winter. El material estudiado responde bien a las descripciones de estos autores.

\section{Cucurbitaria elongata (Fies) Grev.}

Material estudiado: Sierra de las Nieves, Ronda (Málaga). Leg. J.L., 16.VI.1984 (JL 7054). (Fig. 4: A-B-C-D-E).

Hábitat: Sobre ramas muertas de Ulex baeticus.

Observaciones: Los ejemplares estudiados coinciden con la descripción dada por Mirza (1968) para este taxon.

\section{HELOTIALES}

Ascocalyx asiaticus Groves

Material estudiado: Sierra de El Pinar, Grazalema (Cádiz). Leg. M.H., 14.VI.1984 (JL 7057). (Fig. 5: A-B).

Hábitat: Sobre eje principal y ramas superiores de pinsapo muerto, por necrosis basipetal.

Observaciones: El material estudiado concuerda exactamente con los caracteres dados por Groves (1968) para Ascocalyx asiaticus. Solo difieren en el diámetro esporal, ligeramente superior en los ejemplares estudiados por el autor del taxon.

Groves (1968) indica que el estado anamorfo (Bothrodiscus) aparece, a menudo, en el mismo estroma que el teleomorfo. En el material estudiado, procedente de la Sierra de El Pinar, no se ha podido observar este detalle. Esto es debido, posiblemente, a que la fase sexual (apotecios) se desarrolla "sobre tejidos vivos o muertos el mismo año o el anterior; el estado conidial es común a la fase parasítica" (Korf, R., in Ainsworth \& col., 1973).

Se puede pensar, en consecuencia, que se trata de un hongn parásito cuya fase anamorfa puede ser uno de los agentes causantes de la muerte basipetal de los pinsapos.

El hecho de que todos los ascomas observados estuvieran dispuestos en el ápice de los ejes central y ramificaciones (guías de crecimiento del árbol), junto a que todos los pies atacados por este hongo conservan las hojas muertas y hayan tenido necrosis descendente, apoyan esta interpretación.

\section{HYPOCREALES}

Nectria coccinea (Pers.: Fr.) Fr.

Material estudiado: Sierra de las Nieves, Yunquera (Málaga). Leg . J.L., 23.XI.1984 (JL 7083). Cortijo de la Nava de San Luis, Ronda (Málaga). Leg. M.H. \& J.L., 13.VI.1984 (JL 7050). Los Reales, Sierra Bermeja, Estepona (Málaga). Leg. M.H., 13.VI.1984 (JL 7051 ).

Hábitat: Corteza, en rama muerta de Abies pinsapo. 
Observaciones: El material estudiado responde a la descripción dada por Booth (1959) para este taxon.

\section{Nectria fuckeliana Booth}

Material estudiado: Sierra de El Pinar, Grazalema (Cádiz). Leg. G.M. \& J.L., 14.VI.1984 (JL 7045).

Hábitat: Sobre corteza de tronco muerto de Abies pinsapo.

Observaciones: El material estudiado responde fielmente a la descripción dada por Booth (1959) para este taxon. Es típica de coníferas, citada anteriormente sobre Abies (Booth 1959).

Nectria purtonii (Grev.) Berk.

Material estudiado: Cortijo de la Nava de San Luis, Ronda (Málaga). Leg. M.H. 13.VI.1984 (JL 7071) (Fig. 1:H)

Hábitat: Sobre estroma de otro pirenomiceto, en corteza de tronco muerto de Abies pinsapo.

Observaciones: Las ascósporas presentan pequeñas espínulas, lo que concuerda con lo indicado por Samuels (1976), a pesar de ser un carácter no comentado por Booth (1959).

Scoleconectria cucurbitula (Tode: Fr.) Booth

Material estudiado: Pinsapar del Cortijo de la Nava de San Luis, Ronda (Málaga). Leg. M.H., 21.XI.1984 (JL 7089). (Fig. 2: E-F-G-HI) :

Hábitat: Sobre corteza de Abies pinsapo.

Observaciones: Especie siempre citada sobre coníferas. Se caracteriza, principalmente, por presentar 2-4 ascósporas, cilíndrico-claviformes, vermiformes, pluriseptadas, las cuales germinan directamente en el interior del asco. Dan lugar a "ascoconidios hialinos, alantoides, que ocupan todo el lumen del asco" (Booth, 1959).

El género Scoleconectria fué propuesto por Seaver, en 1909, para aquellas especies de Ophionectria Saccardo que presentan peritecios dispuestos sobre estroma (Booth, 1959). Hawksworth \& col. (1983) consideran el taxon de Seaver como sinónimo de Ophionectria. Sin embargo, Rogerson (1970) es partidario de mantener separados ambos taxones. Por nuestra parte, de acuerdo con Rogerson (1970), consideramos como válido el binomio Scoleconectria cucurbitula (Tode: Fr.) Booth, 1959 .

Este taxon había sido citado anteriormente para la provincia de Málaga, como Nectria cucurbitula Fr., por Benito \& Torres (1965).

\section{OSTROPALES}

Ostropa cinerea (Pers.) Fr.

Material estudiado: Sierra de El Pinar, Grazalema (Cádiz). Leg. M.H., 14.XI.1983 (JL 7066).

Hábitat: Sobre rama muerta, corteza y madera, de Quercus faginea.

Observaciones: Género monoespecífico, caracterizado por la presencia de una papila subglobosa, erumpente, provista de una hendidura longitudinal. Ascos y ascósporas son muy parecidos a los representantes 
de la familia Strictidaceae. Por esta razón, Ostropa cinerea ha sido transferido a dicha familia (Sherwood, 1977 in Eriksson, 1981).

\section{PEZIZALES}

Sarcoscypha coccinea (Fr.) Lambotte

Material estudiado: Sierra de El Pinar, Grazalema (Cádiz). Leg. M.H. \& R. Pardo, 27.III.1983 (MH 3466).

Hábitat: Talud quemado, con Rubus sp., en el pinsapar.

Observaciones: Especie banal, fácilmente reconocible por sus apotecios de color rojo sangre. El material estudiado coincide con la descripción dada por Dennis (1978), para este taxon.

\section{SPHAERIALES}

\section{Zignoëlla fallax Saccardo}

Material estudiado: Sierra de El Pinar, Grazalema (Cádiz). Leg. J.L., 14.VI.1984 (JL 7056). (Fig. 3: F).

Hábitat: Sobre tronco descortezado de Hedera helix.

Observaciones: El material responde a la descripción dada por Munk (1957), el cual incluye el género Zignoëlla Saccardo, en la tribu Rhamphorioideae, de la familia Diaporthaceae (Diaporthales). Ainsworth \& col. (1973) mantienen este criterio. Barr (1976) considera el taxon correspondiente a la familia Trichosphaeriaceae del orden Xylariales (Barr, 1978). Por su parte, Dennis (1978) mantiene el género en la familia Trichosphaeriaceae, del orden Sphaeriales. De igual modo se manifiestan Hawksworth \& col. (1983), al incluir este género en el orden Sphaeriales.

\section{Coniochaeta malacotricha (Auersw.) Traverso}

Material estudiado: Sierra de las Nieves, Ronda (Málaga). Leg. J.L.; 13.VI.1984 (JL 7046). Sierra de las Nieves, Yunquera (Málaga). Leg. J.L., 23.XI.84 (JL 7080). (Fig. 6: A-B-C-D-E).

Hábitat: Sobre rama descortezada de Abies pinsapo.

Observaciones: Taxon fácilmente reconocible por sus peritecios de 250$350 \mu \mathrm{m}$ de diámetro, de color negro, provistos de pelos cortos (20-40 $\times 2,5-3,5 \mu \mathrm{m})$, rígidos, divergentes, de color pardo oscuro, con base ensanchada, hasta $6 \mu \mathrm{m}$ de diámetro en la base, y ápice obtuso. Hama tecio formado por paráfisis filiformes, de mayor longitud que los ascos, de 1,5-2,5 $\mu \mathrm{m}$ de diámetro. Ascos octosporados, de 110-130 x 1011(12) $\mu \mathrm{m}$, cilíndricos, pedicelados, unitunicados. Ascósporas de 12-

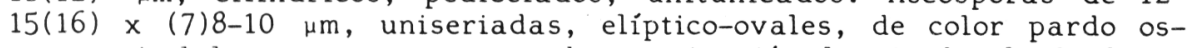
curo, unicelulares, con ranura de germinación longitudinal, hialina. Especie típica de coniferas, citada por Traverso (1906) sobre Abies.

Xylaria hypoxylon (L. ex Hooker) Grev.

Material estudiado: Pinsapar del Cortijo de la Nava de San Luis, Ronda (Málaga). Leg. M.H., 22.XI.1984 (JL 7078).

Hábitat: Sobre tocón de Quercus rotundifolia. 

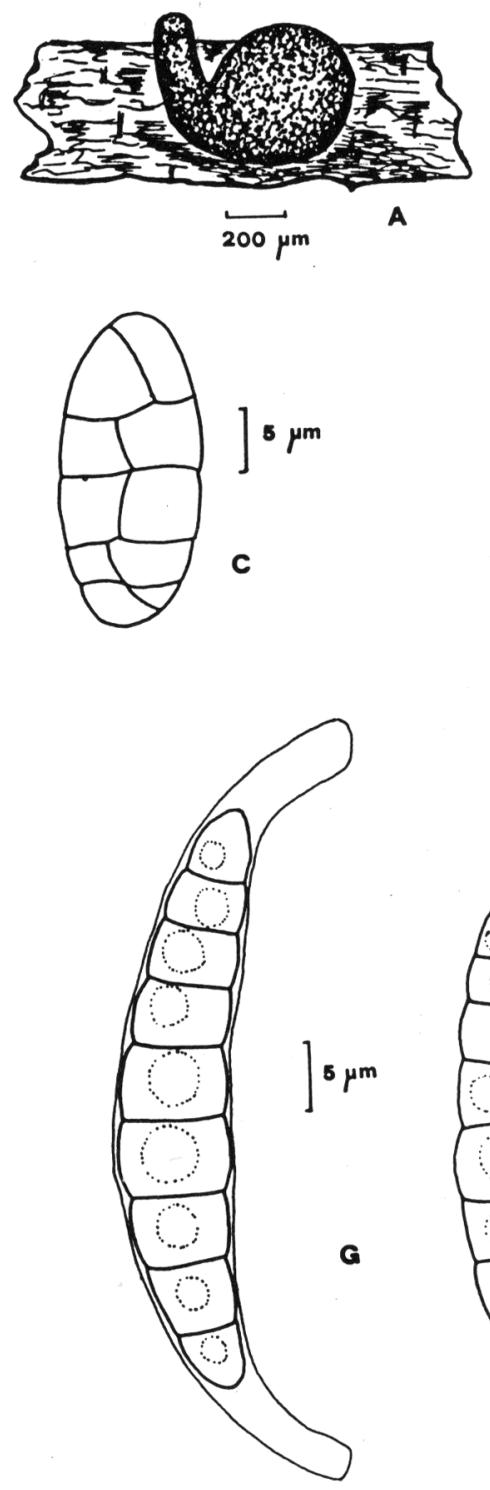

(ब)

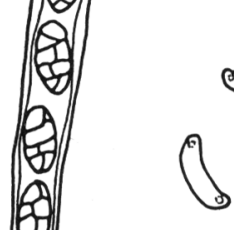

S

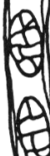

$\int 10 \mu \mathrm{m}$<smiles>[C]1CCCCC1</smiles><smiles>C1CCCCC1</smiles><smiles>[C+]1C=CC=C1</smiles>

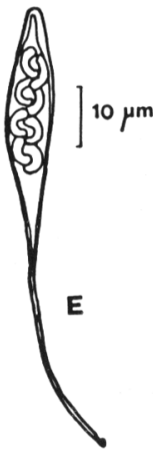

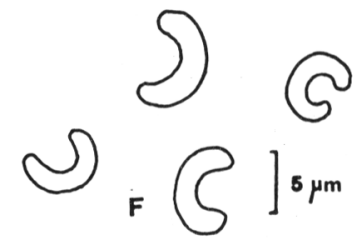

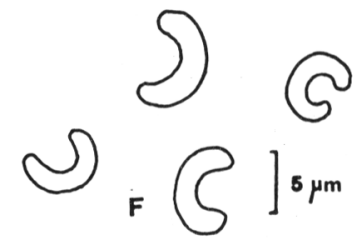

B
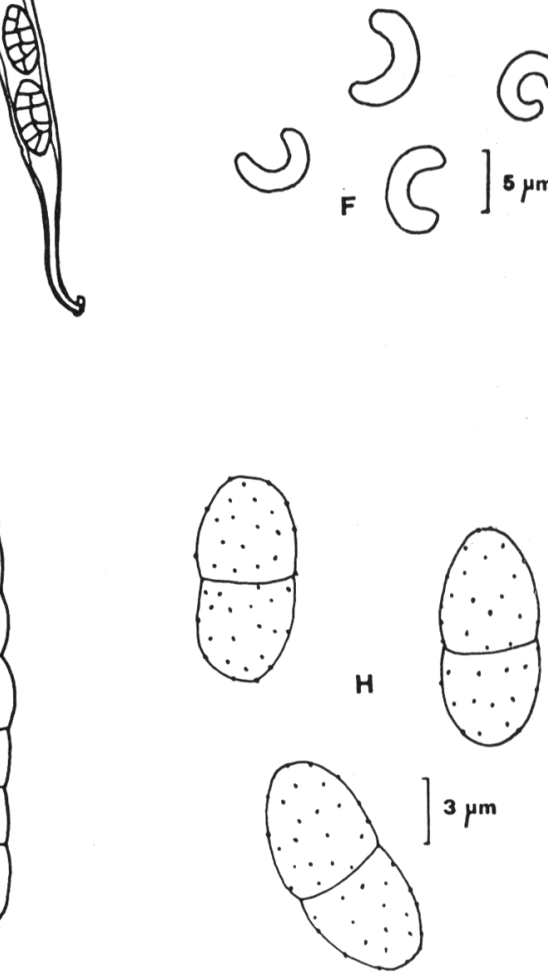

Figura 1.- Rhamphoria obliqua: A. peritecio, B. asco con ascósporas, C. ascósporas. Eutypa flavovirescens: D. ascósporas. E. polymorpha subsp. cyclospora: E. asco con ascósporas, F. ascósporas. Lophiostoma alpigenum: G. ascósporas. Nectria purtonii: H. ascósporas. 


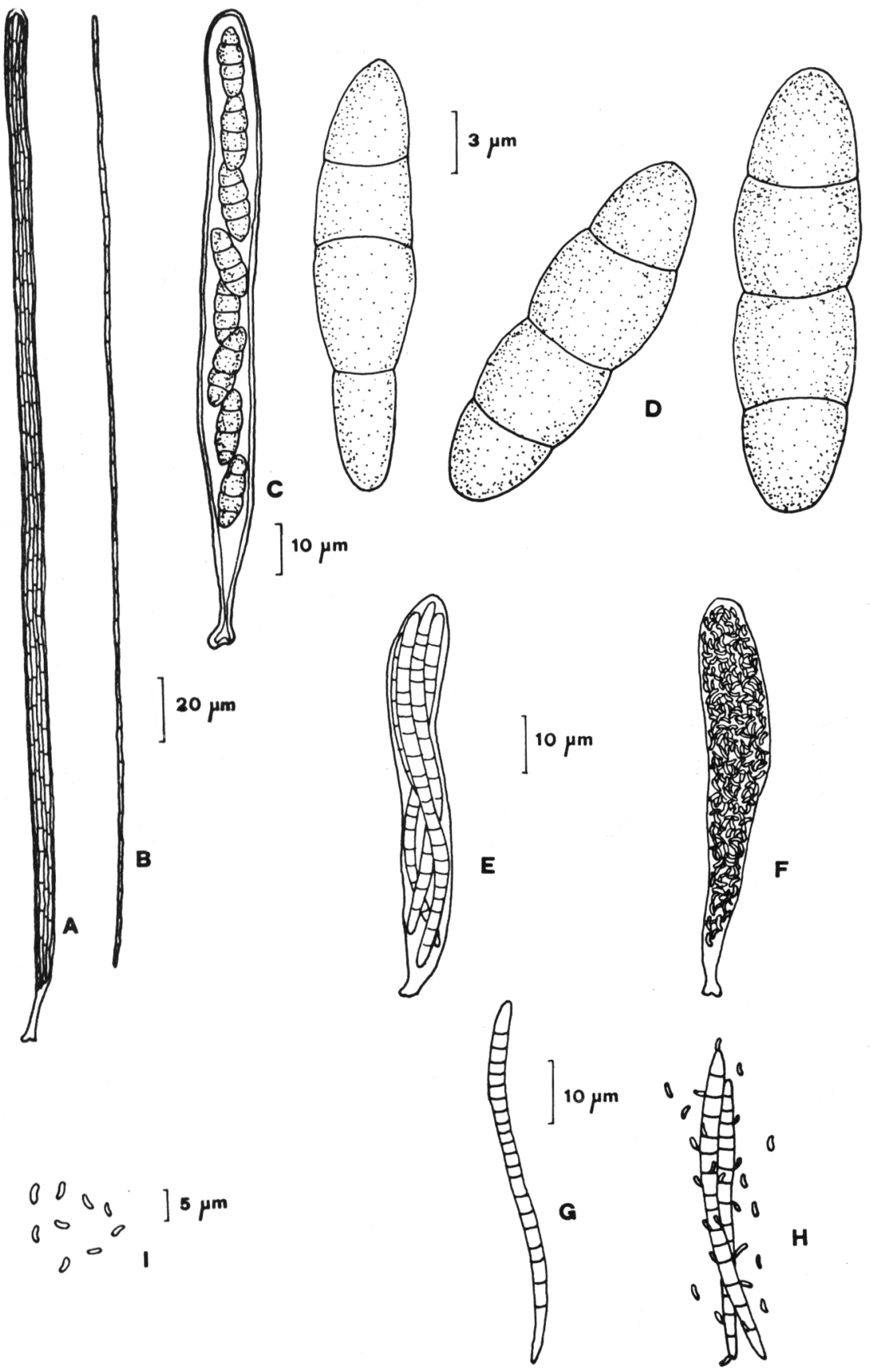

Figura 2.- Lophium mytilinum: A. asco con ascósporas, B. ascósporas. Mytilidion tortile: C. asco con ascóspora, D. ascósporas Scoleconectria cucurbitula: E. asco con ascósporas, F. asco con ascoconidios, G. ascósporas, H. ascóspora originando ascoconidios, I. ascoconidios. 

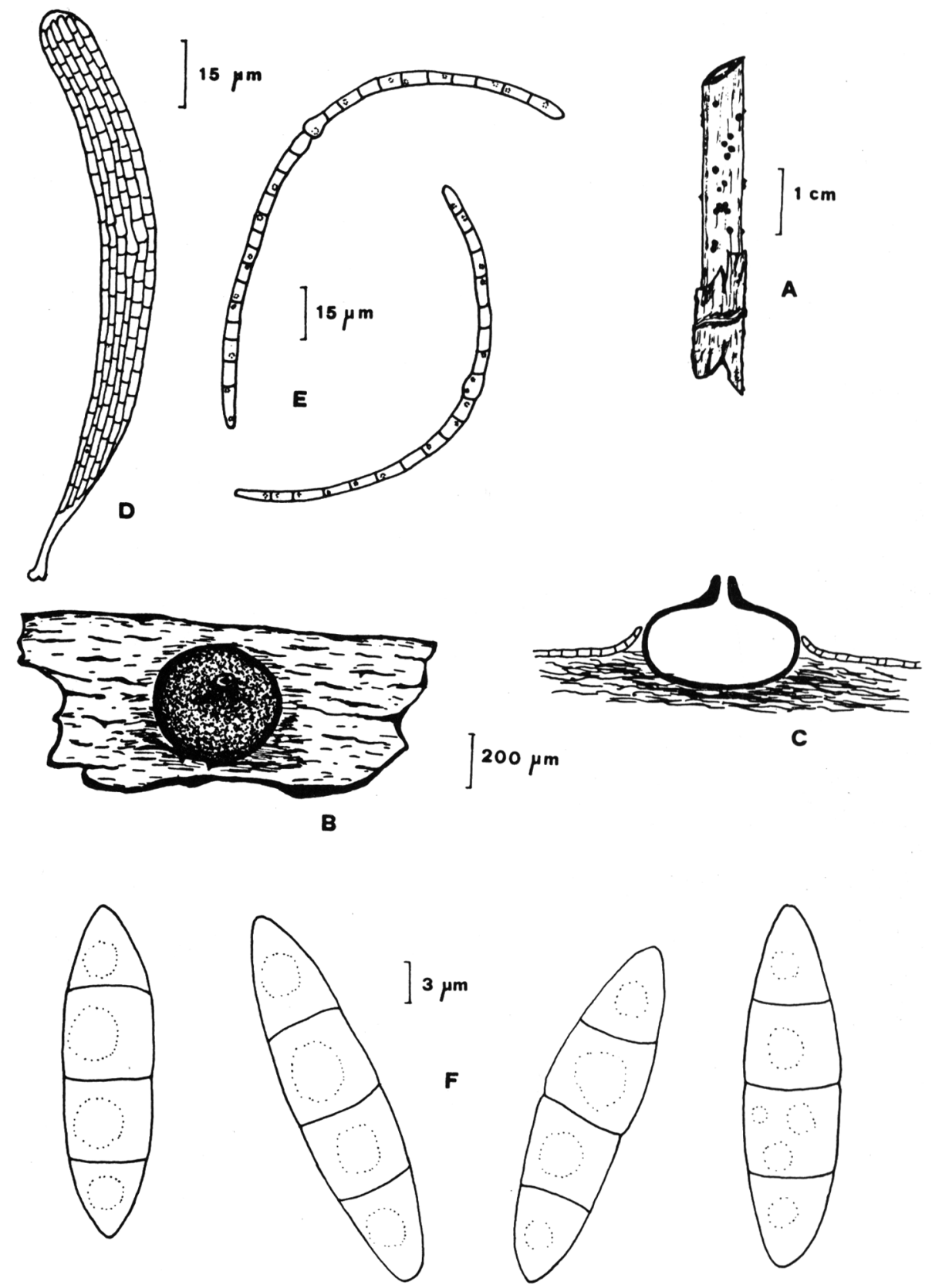

Figura 3.- Ophiobolus erythrosporus: A. aspecto general de los pseudotecios sobre el sustrato, B. pseudotecio, C. detalle de un corte transversal del pseudotecio, D. asco con ascósporas, E: ascósporas. Zignoëlla fallax: F. ascósporas. 

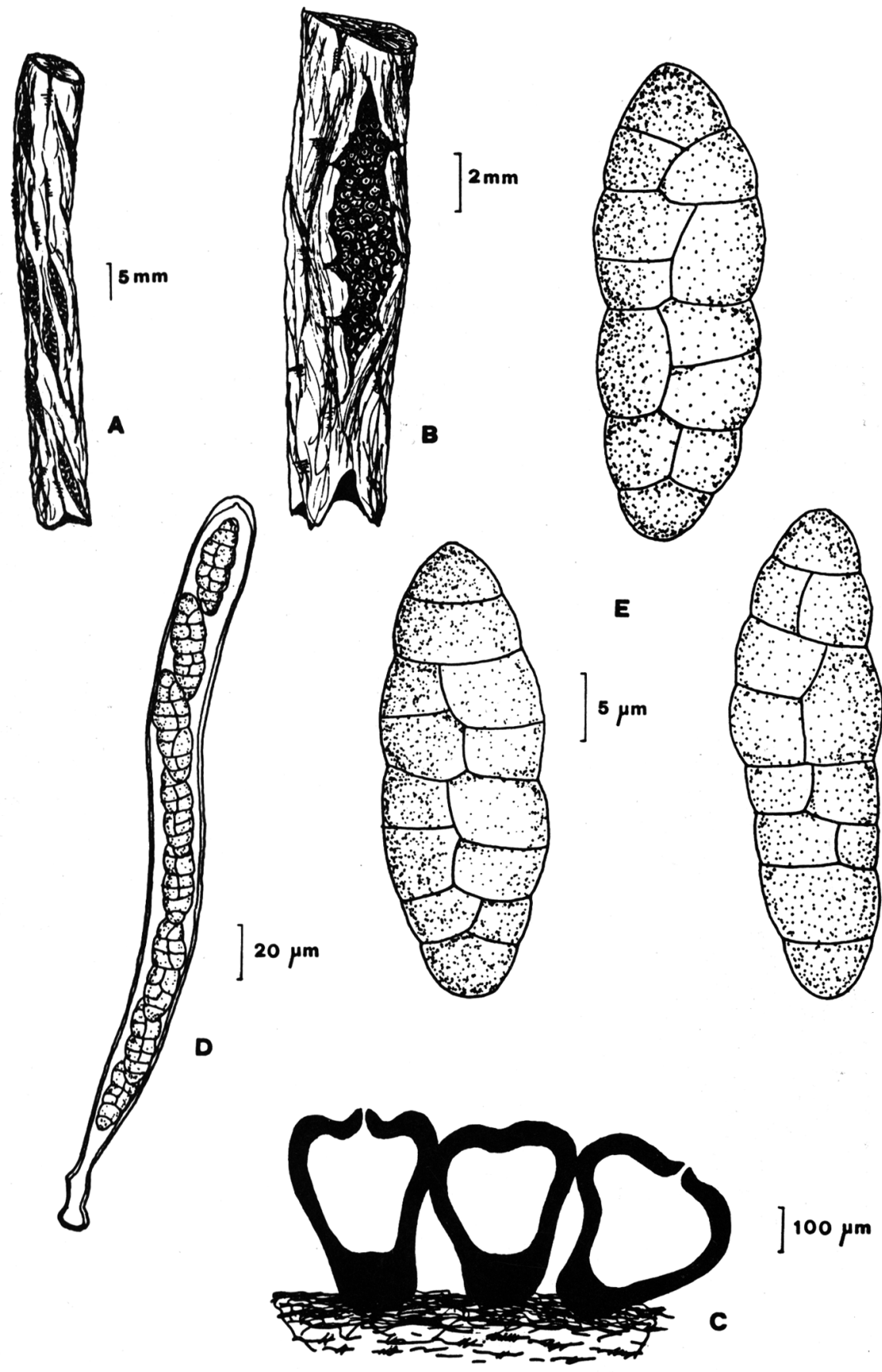

Figura 4.- Cucurbitaria elongata: A-B. aspecto general de los estromas sobre el sustrato, C. detalle de un corte transversal de los pseudotecios, D. asco con ascósporas, E. ascósporas. 


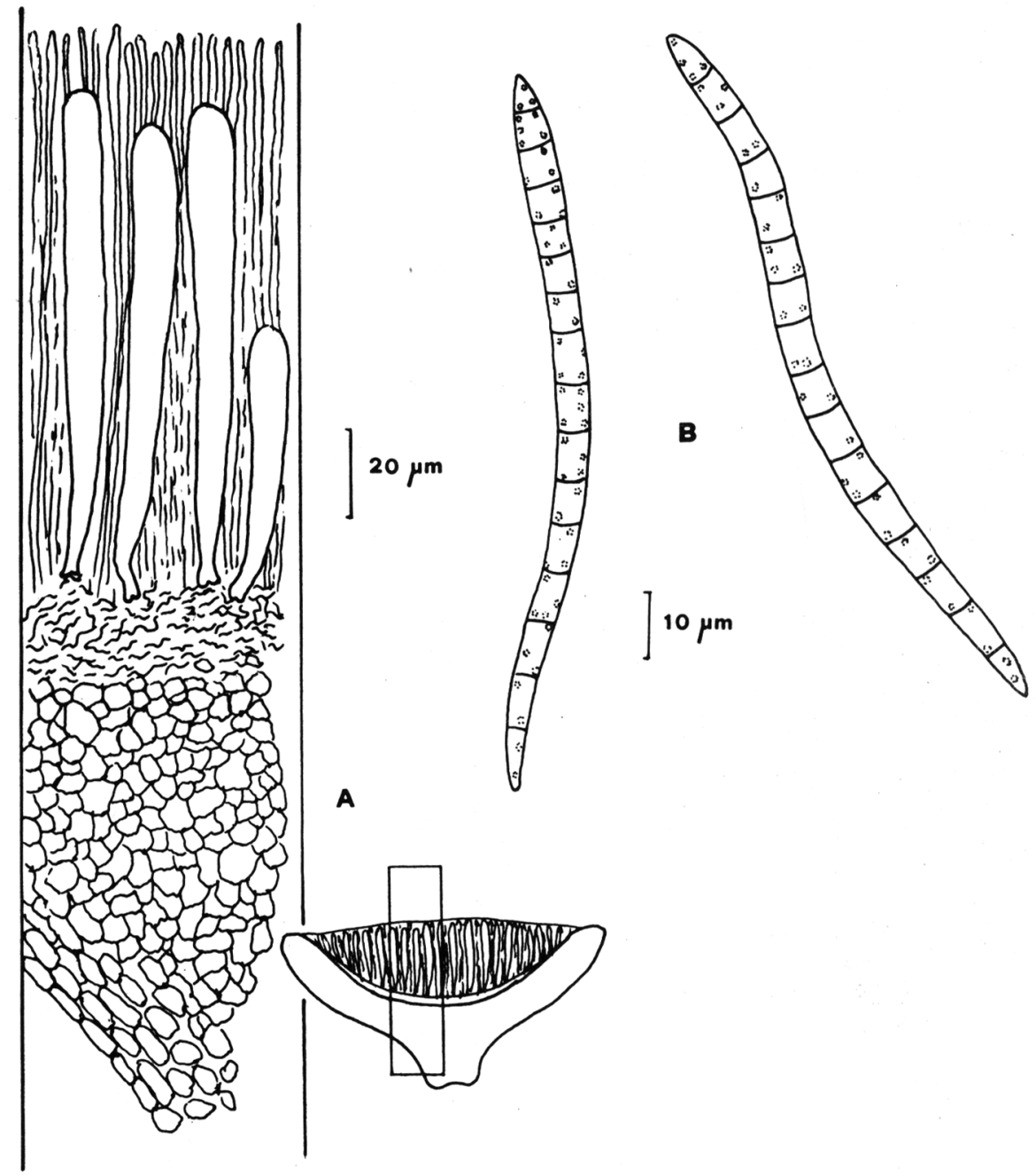

Figura 5.- Ascocalyx asiaticus. A. detalle de un corte transversal de un apotecio, B. ascóspora. 

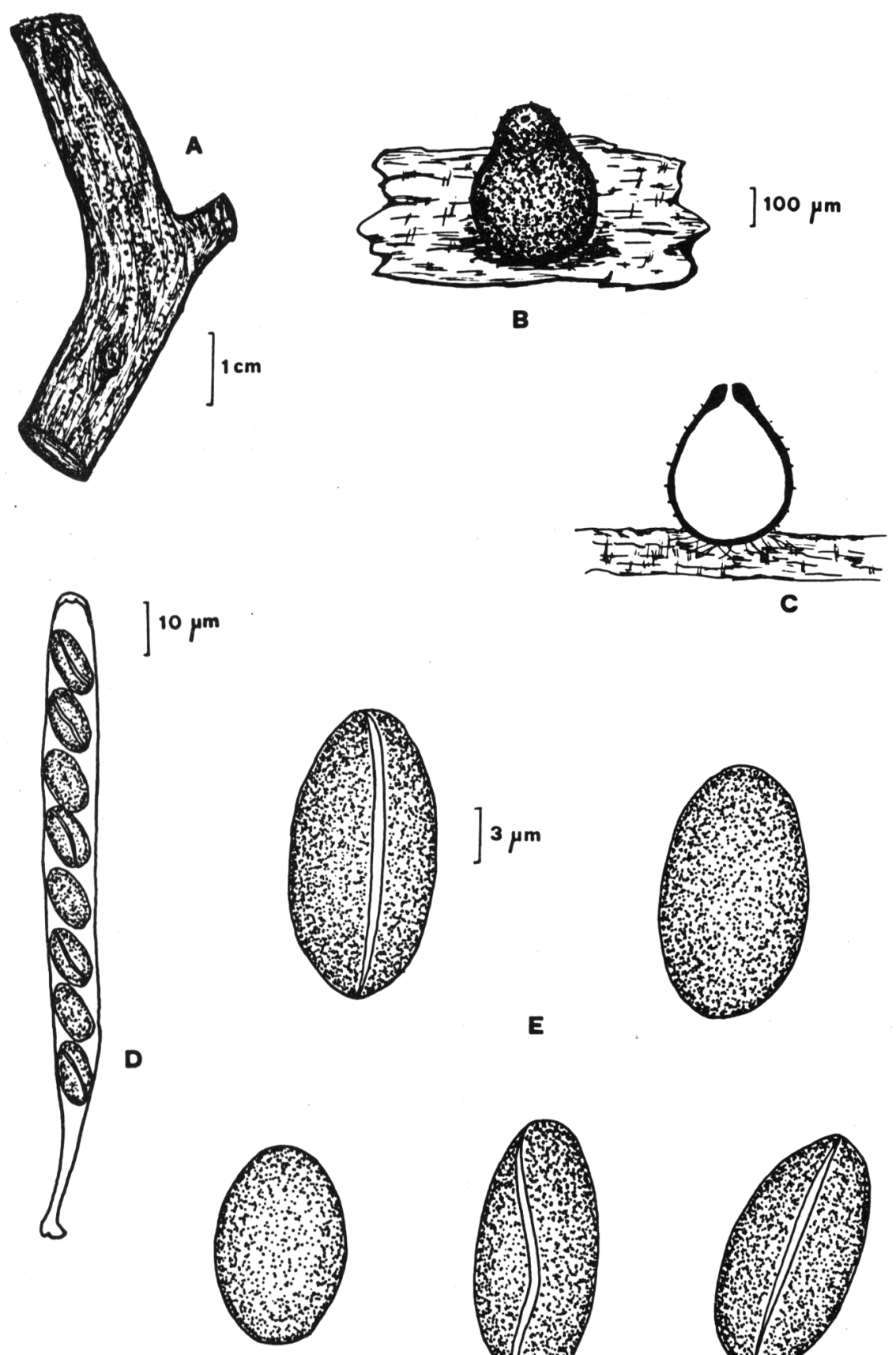

C
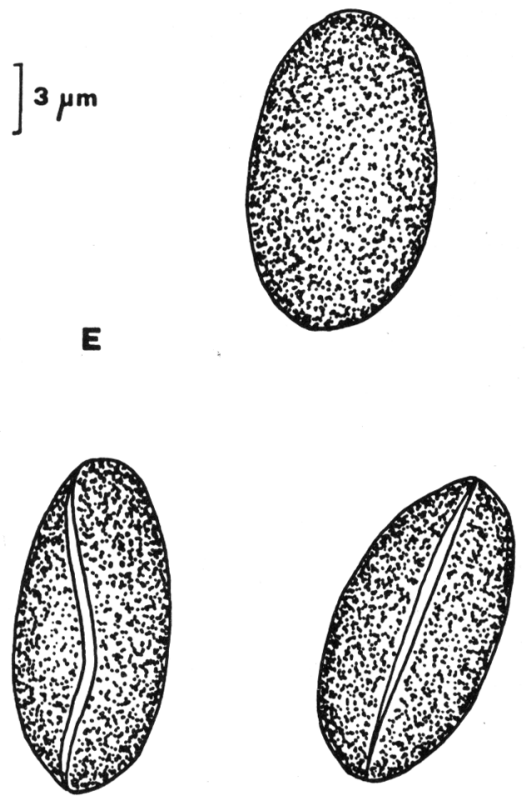

Figura 6.- Coniochaeta malacotricha: A. aspecto general de los peritecios sobre la madera, B. peritecio, C. detalle de un corte transversal del peritecio, D. Asco con ascósporas, E. ascósporas. 


\section{BIBLIOGRAFIA}

AINSWORTH, G.C., SPARROW, F.K. SUSSMAN, A.S. -1973- The Fungi. Vol. IV. A. Academic Press. New York.

ARX, J.A. VON \& MULLER, E. -1954- Die Gattungen der Amerosporen Pyrenomyceten. Beitr. Krytogamenfl. Schweiz.

ARX, J.A. VON \& MULLER, E. -1975- A re-evaluation of the bitunicate Ascomycetes with keys to families and genera. Stud. Mycol'., 9: $159 \mathrm{pp}$.

BARR, M.E. -1976- Perspectives in the Ascomycotina. Mem. New York. Bot. Gard., 28: 1-8.

BARR, M.E. -1978- The Diaporthales in North America. Mycologia memoir 7. J . Cramer. New York.

BENITO MARTINEZ, J. \& TORRES JUAN, J. -1965- Enfermedades de los coníferos españoles. Inst. Forest. Invest. y Exp., 88.

BERLESE, A.N. -1890-1905- Icones Fungorum. Vol. I-III. (reprint 1968). J. Cramer, New York.

BERTAULT, R. -1974- Contribution a la Flore Mycologique de l'Andalousie. Collectanea Botanica, 9(2): 25-44.

BOUTH, C. -1959- Studies of Pyrenomycetes, IV. Nectria (part.1). Mycological Papers, 73-115 pp.

CHADEFAUD, M. -1960- Traité de botanique systématique I. Les végétaux non vasculaires (Cryptogamie). Masson. Paris.

CHECA, J. \& MORENO, G. -1984- Estudios sobre Pyrenomycetes y Loculoascomycetes (Ascomycotina). I. Bol. Soc. Micol. Castellana. 8: 15-22.

CHESTERS, C.G.C. \& BELL, A. -1970- Studies in the Lophiostomataceae Sacc. Mycological Papers, 120.

DENNIS, R.W.G. -1978- British Ascomycetes. J. Cramer Vaduz.

ELLIS, J.B. EVERHART, B.M. -1892- The North American Pyrenomycetes (reprint 1966). Johnson repr. Corporation. New York.

ERIKSSON, O. -1981- The families of bitunicate Ascomycetes. Opera botanica, 60: 1-220.

GROVES, J.W. -1968- Two new species of Ascocalyx. Canad. Journ. of Bot., 46(10): 1273-1278.

HAWKSWORTH, D.L., SUTTON, B.C. \& AINSWOTH, G.C. -1983- Dictionary of the Fungi. Surrey .

HOLM, L. -1957-Etudes taxonomiques sur les Pléosporacées. Symb. Bot. Ups. 14: 3. Uppsala.

HONRUBIA, M., LARIOS, J.M. \& GALLEGO, E. Notas sobre Ascomycetina en el S.E. de España Peninsular. Anal. J. Bot. En prensa.

LUTTRELL, E.S. -1951- Taxonomy of the Pyrenomycetes. Univ. Missouri Studies, 24: 1-120.

MALENCON, G. -1968- Contribution à la Flore Muycologique de l'Andalousie. Collectanea Botanica, 7(2): 707-725.

MALENCON, G. \& BERTAULT, R. -1976- Champignons de la Péninsule Ibérique V. Catalogne, Aragon, Andalousie. Acta Phytotax. Barcinon., 19: $1-68$.

MANJON, J.L. \& MORENO, G. -1981- Estudios sobre Aphyllophorales. III. Fructificaciones en Abies pinsapo. Boiss. Cryptogamie, 4(2): 145157.

MIRZA, F. -1968- Taxonomic investigations on the Ascomycetous. Genus Cucurbitaria S.F. Gray. Nova Hedwigia, 16: 161-213.

MULLER, E. \& ARX, J.A. VON -1962- Die Gattungen der Didymosporen Pyrenomyceten. Beitr. Kryptogamenfl. Schweiz., 11(2): 1-922.

MULlER, E. \& ARX, J.A. VON -1979- in AINSWOTH, G.C. \& al. The Fungi. Vol. IV A. pp. 87-132. Academic Press. London.

MUNK, A. -1953- The system of Pyrenomycetes. Dansk. Bot. Arkiv. 15, 2. $163 \mathrm{pp}$. Copenhagen.

MUNK, A. -1957- Danish Pyrenomycetes. Dansk. Bot. Arkiv. 17. 1-491 pp.

ROGERSON, C.T. -1970- The Hypocrealean Fungi (Ascomycetes, Hypocreales) Mycologia, 62(5): 865-910. 
SACCARDO, P. -1883- Syll loge Fungorum. Vol. 2.

SAMUELS, G.J. -1976- A revision of the Fungi formerly classified as Nectria subgenus Hyphonectria. Mem. of the New York Bot. Garden, 26 (3) : $1-126$.

SEAVER, F.K. -1909- The Hypocreales of North America. Mycologia, 1: 4576.

SIVANESAN, A. -1984- The Bitunicate Ascomycetes. J. Cramer.

TRAVERSO, J.B. -1906- Flora Italica Cryptogama.

ZOGG, H. -1962- Die Histeriaceae und Lophiaceae. Beitr. Kryptogammenfl. Schweiz., 11(3): 1-190. 